\title{
Natural variations in flavonoids and triterpenoids of Cyclocarya paliurus leaves
}

\author{
Caowen Sun ${ }^{1,2} \cdot$ Xulan Shang ${ }^{1,2} \cdot$ Haifen Ding ${ }^{1} \cdot$ \\ Yanni $\mathrm{Cao}^{1} \cdot$ Shengzuo Fang ${ }^{1,2}$
}

Received: 9 July 2019 / Accepted: 5 March 2020 / Published online: 11 May 2020

(C) The Author(s) 2020

\begin{abstract}
Cyclocarya paliurus is widely distributed in subtropical areas of China. Secondary metabolites in the leaves, including flavonoids and triterpenoids, provide protection against diseases such as hyperlipidemia, diabetes, hypertension, and hypoimmunity. In this study, 343 C. paliurus leaves were collected from 32 distinct populations covering most of the main distribution, to deduce the response of flavonoids and triterpenoids to seven environmental factors. Principal component analysis was performed using seven flavonoid and six triterpenoid compounds. The results show that altitude, latitude, and longitude significantly contributed to variations in total flavonoid and triterpenoid contents. In addition, the interaction of these metabolites was extensively correlated with environment changes, and therefore should be considered during selection and cultivation.
\end{abstract}

Caowen Sun and Xulan Shang have contributed equally to this work.

Project funding: This work was supported by the Natural Science Foundation of Jiangsu Province (Grants No. BK 20160926), the Forestry Science and Technology Promotion Project from the State Forestry Administration of China [2017(08)], and the Priority Academic Program Development of Jiangsu Higher Education Institutions (PAPD).

The online version is available at https://www.springerlink.com.

Corresponding editor: Yanbo Hu.

Shengzuo Fang

fangsz@njfu.edu.cn; fangsz@njfu.com.cn

1 College of Forestry, Nanjing Forestry University, Nanjing 210037, People's Republic of China

2 Co-Innovation Center for Sustainable Forestry in Southern China, Nanjing Forestry University, Nanjing 210037, People's Republic of China
Keywords Cyclocarya paliurus - Environmental factors · Flavonoids $\cdot$ Secondary metabolite variation $\cdot$ Triterpenoids

\section{Introduction}

Cyclocarya paliurus (Batal) Iljnsk. is distributed across subtropical areas of China. The leaves have long been used to make a traditional sweet tea (Shu et al. 1995a, b; Fang et al. 2006). This species was investigated as a substitute to the common tea from Camellia sinensis (L.) Kuntze and was found to be beneficial to human health ( $\mathrm{Li}$ et al. $2017 \mathrm{a}, \mathrm{b})$. The leaves have high concentrations of secondary metabolites that may be protective against a range of diseases, including hyperlipidemia, diabetes, and hypertension (Kurihara et al. 2003; Xie et al. 2006; Wang et al. 2017a; Li et al. 2017a, b). Previously, six triterpene compounds were isolated from the leaves by HPLC (high performance liquid chromatography): arjunolic acid, cyclocaric acid B, pterocaryoside B, pterocaryoside A, hederagenin, and oleanolic acid (Shu et al. 1995a, b; Wright et al. 2014; Shang et al. 2015; Cao et al. 2017; Liu et al. 2018). Seven flavonoid compounds have also been isolated, including quercetin-3-Oglucuronide, quercetin-3-O-galactoside, isoquercitrin, kaempferol-3-O-glucuronide, kaempferol 3-O-glucoside, quercetin-3-O-rhamnoside, and kaempferol-3-O-rhamnoside (Shang et al. 2015; Xie et al. 2015; Cao et al. 2017, 2018; Liu et al. 2018). Many studies have examined flavonoids and triterpenoids because of their health-promoting functions (Shu et al. 1995a, b; Xie et al. 2006, 2015; Li et al. 2017a, b).

Secondary metabolite biosynthesis in leaves are influenced by intrinsic and extrinsic factors (Szakiel et al. 2011). Intrinsic factors include genetic, genotypic and physiological status such as species, provenances, populations, families, plant growth stages and carbon-nitrogen metabolism. 
Extrinsic factors include biotic and abiotic environmental stimuli, such as reacting to pathogens, parasites, insects, animals (Szakiel et al. 2011), and light, soil carbon and temperature (Barnes et al. 2015). Generally, geographical variation research on secondary metabolites focuses on environmental factors along with genotype variations. These research programs select the main effects at work in a geographical area from comprehensive factors (Fang et al. 2011; Li et al. 2017a, b; Cao et al. 2018; Deng et al. 2018, 2019a, b). As a result, breeding and cultivation research is based on this information.

Considerable efforts are currently focused on genotypic and environmental interactions of $C$. paliurus germplasm secondary metabolite variation (Fang et al. 2006, 2011, Fang and Fu 2007; Deng et al. 2012, 2014, 2017; Li et al. 2017a, b). The research has made progress at the population level. Liu et al. (2018) found that geographical location significantly affects the phenolic composition of $C$. paliurus, and Zhou et al. (2019) noted that the content of total flavonoids and total polyphenols from 21 natural populations varied significantly. Deng et al. (2019a, b) reported that individual triterpenoids were primarily influenced by genotype, while the environment accounted for variations in total triterpenoid content. Cao et al. (2018) determined that, at the family level, flavonoid content variations of $C$. paliurus were significant. This has made it important to study secondary metabolite variations based on geography at the family level. More research has focused on variations of phenols, terpenoids and other secondary metabolites separately (Fang et al. 2011; Deng et al. 2012, 2014, 2015; Liu et al. 2018; Cao et al. 2018); limited information is available on the competing processes of flavonoid and triterpenoid accumulations. This has made it difficult to select beneficial plant traits and has impeded breeding and cultivation. Additional research on the geographical variations of $C$. paliurus secondary metabolites is therefore required. In this study, secondary metabolite variations in C. paliurus germplasm was investigated over a wide distribution area. The objectives were to analyze flavonoid and triterpenoid variation patterns in leaves of naturally occurring plants at the family level, and to investigate relationships between secondary metabolites and environmental factors. The results will be important for targeting directed breeding approaches and for the identification of suitable areas.

\section{Materials and methods}

\section{Plant material}

Leaves of C. paliurus were collected from a total of 343 trees at 32 distinct localities covering most of the $C$. paliurus range in China in 2014. Samples were collected from 12 provinces, Anhui, Zhejiang, Fujian, Shanxi, Henan, Hunan, Hubei, Jiangxi, Guangxi, Guizhou, and Sichuan (Fig. 1), across a wide area of southern China at $9.0^{\circ}$ latitude, $17.4^{\circ}$ longitude, and $1370 \mathrm{~m}$ a.s.l. (Table 1). Only mature leaves were collected in October to ensure that secondary metabolites were fully developed. The trees were mainly dominant or subdominant greater than 20 years old. Samples were dried, crushed, and stored at room temperature prior to analysis (chemical analysis was carried out in 2014).

\section{Flavonoid and triterpenoid determination}

Approximately $75 \mathrm{~g}$ samples from each location were extracted with $80 \%$ ethanol before being incubated in a water bath at $90{ }^{\circ} \mathrm{C}$ for $2 \mathrm{~h}$. This was followed by shaking for $15 \mathrm{~min}$ and centrifugation at $8000 \mathrm{r} / \mathrm{min}$ for $15 \mathrm{~min}$. Supernatants were concentrated at $40{ }^{\circ} \mathrm{C}$ to obtain the extracts.

Flavonoid and triterpenoid contents were analyzed using high-performance liquid chromatography (HPLC) (Liu et al. 2018). Extracts were filtered through a $0.4-\mu \mathrm{m}$ polytetrafluoroethylene filter before HPLC analysis (Agilent 1200
Fig. 1 Sampling locations of $C$. paliurus

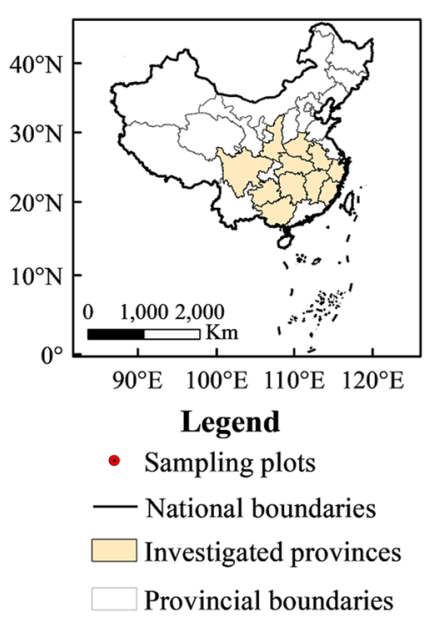

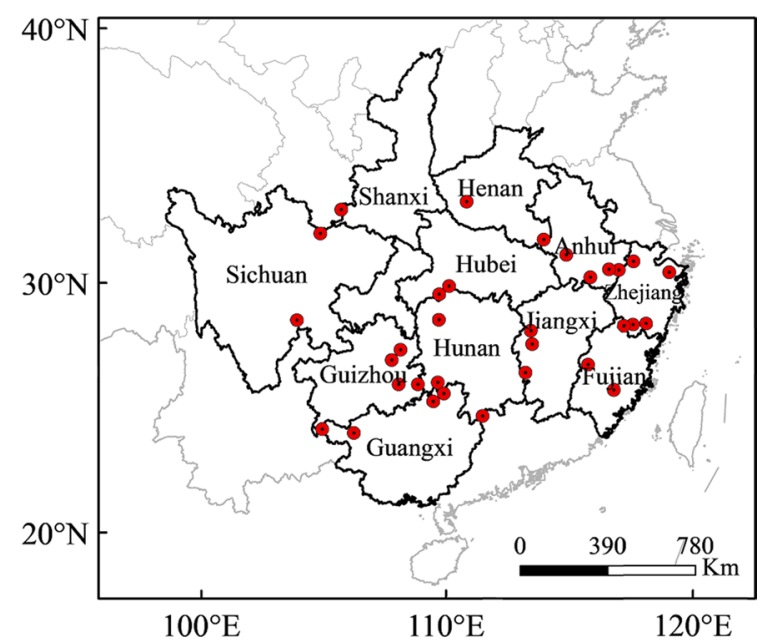


Table 1 Collection locations and associated environmental factors

\begin{tabular}{|c|c|c|c|c|c|c|c|c|}
\hline Location & Latitude $\left({ }^{\circ} \mathrm{N}\right)$ & Longitude $\left({ }^{\circ} \mathrm{E}\right)$ & Altitude (m) & $\begin{array}{l}\text { Annual mean } \\
\text { temperature } \\
\left({ }^{\circ} \mathrm{C}\right)\end{array}$ & $\begin{array}{l}\text { Annual mean } \\
\text { rainfall }(\mathrm{mm})\end{array}$ & $\begin{array}{l}\text { Annual } \\
\text { sunlight } \\
\text { (h) }\end{array}$ & $\begin{array}{l}\text { Frost-free } \\
\text { days (day) }\end{array}$ & Sample size \\
\hline Jixi County, Anhui & 30.15 & 118.89 & 730 & 12.1 & 1726 & 1920 & 233 & 11 \\
\hline Jingde County, Anhui & 30.23 & 118.45 & 610 & 13.8 & 1657 & 1784.1 & 240 & 3 \\
\hline Qimen County, Anhui & 30.02 & 117.53 & 400 & 13.9 & 1775 & 1669 & 232 & 5 \\
\hline Shucheng County, Anhui & 31.02 & 116.54 & 770 & 12.3 & 1606 & 1969 & 224 & 12 \\
\hline Ningbo City, Zhejiang & 29.76 & 121.22 & 715 & 14 & 1527 & 1850 & 232 & 8 \\
\hline Longquan City, Zhejiang & 27.91 & 119.19 & 1200 & 11.8 & 2119 & 1849.8 & 263 & 7 \\
\hline $\begin{array}{l}\text { Wencheng County, } \\
\text { Zhejiang }\end{array}$ & 27.88 & 119.79 & 915 & 13.9 & 1913 & 1764.4 & 285 & 5 \\
\hline Anji County, Zhejiang & 30.41 & 119.64 & 540 & 13.4 & 1472 & 1666 & 231 & 7 \\
\hline Pucheng County, Fujian & 27.93 & 118.76 & 930 & 15.8 & 1998 & 1900 & 254 & 10 \\
\hline Quanzhou City, Fujian & 25.43 & 117.93 & 500 & 18.1 & 1593 & 1908 & 320 & 3 \\
\hline Mingxi County, Fujian & 26.57 & 116.93 & 564 & 17.4 & 1821 & 1788.6 & 261 & 12 \\
\hline Lueyang County, Shanxi & 33.36 & 105.87 & 1200 & 13 & 714 & 1558.3 & 236 & 9 \\
\hline Nanzhao County, Henan & 33.48 & 112 & 880 & 11.5 & 834 & 1850.5 & 219 & 8 \\
\hline $\begin{array}{l}\text { Shangcheng County, } \\
\text { Henan }\end{array}$ & 31.72 & 115.55 & 920 & 12.2 & 1368 & 1763.1 & 222 & 6 \\
\hline $\begin{array}{l}\text { Yongshun County, } \\
\text { Hunan1 }\end{array}$ & 28.88 & 110.33 & 670 & 14.8 & 1492 & 1306 & 286 & 9 \\
\hline Shaoyang City, Hunan & 26.37 & 110.13 & 1000 & 16.7 & 1320 & 1348.9 & 304 & 16 \\
\hline $\begin{array}{l}\text { Yongshun County, } \\
\text { Hunan2 }\end{array}$ & 24.92 & 112.03 & 845 & 17.4 & 1502 & 1758 & 308 & 10 \\
\hline Hefeng County, Hubei & 29.88 & 110.42 & 1125 & 11.3 & 1499 & 1342 & 245 & 6 \\
\hline Wufeng County, Hubei & 30.19 & 110.9 & 969 & 12.6 & 1650 & 1533 & 240 & 15 \\
\hline Xiushui County, Jiangxi & 28.16 & 114.52 & 827 & 13.5 & 1655 & 1600.4 & 247 & 15 \\
\hline $\begin{array}{l}\text { Jinggangshan County, } \\
\text { Jiangxi }\end{array}$ & 26.51 & 114.1 & 967 & 13.2 & 1816 & 1511 & 241 & 10 \\
\hline Fenyi County, Jiangxi & 27.63 & 114.53 & 565 & 15.3 & 1691 & 1251 & 270 & 14 \\
\hline Baise City, Guangxi1 & 24.46 & 106.34 & 1448 & 15.6 & 1364 & 1906.6 & 357 & 12 \\
\hline Baise City, Guangxi2 & 24.61 & 104.95 & 1770 & 16.7 & 1212 & 1569.3 & 357 & 11 \\
\hline $\begin{array}{l}\text { Longsheng County, } \\
\text { Guangxi }\end{array}$ & 25.62 & 109.89 & 606 & 14.5 & 1629 & 1244 & 314 & 11 \\
\hline Ziyuan County, Guangxi & 25.92 & 110.38 & 850 & 15.5 & 1580 & 1275 & 300 & 10 \\
\hline Liping County, Guizhou & 26.34 & 109.24 & 727 & 16.1 & 1311 & 1317.9 & 277 & 11 \\
\hline Jianhe County, Guizhou & 26.37 & 108.38 & 1240 & 15.1 & 1265 & 1236.3 & 300 & 15 \\
\hline Shiqian County, Guizhou & 27.35 & 108.11 & 1239 & 13.3 & 1256 & 1232.9 & 316 & 16 \\
\hline Yinjiang County, Guizhou & 27.74 & 108.51 & 1032 & 14.5 & 1231 & 1255 & 300 & 7 \\
\hline Muchuan County, Sichuan & 28.97 & 103.78 & 1200 & 17.3 & 1332 & 968 & 332 & 34 \\
\hline Guangyuan City, Sichuan & 32.42 & 104.86 & 1570 & 14.1 & 1027 & 1292 & 243 & 12 \\
\hline
\end{tabular}

series HPLC system, Waldbronn, Germany). A Waters 2489 ultraviolet detector (Waters, Milford, MA, USA) and an X-Bridge $\mathrm{C} 18$ column $(250 \times 4.6 \mathrm{~mm})$ were used. Eluent A was water containing $0.01 \%$ formic acid while eluent B was acetonitrile with $0.01 \%$ formic acid. The ratio of $\mathrm{A}$ to $\mathrm{B}$ in stepwise elution was: 0-13 $\mathrm{min}, 92: 8(\mathrm{v} / \mathrm{v}) ; 13-28 \mathrm{~min}$, $81: 19$; 28-42 min, 79:21; 42-60 min, 50:50; 60-64 min, 55:45; 64-74 min, 44:56; 74-90 min, 34:66; 90-95 min, 15:85; and 95-100 min, 0:100 (Liu et al. 2018). The flow rate was $1.0 \mathrm{ml} / \mathrm{min}$, the injection volume $10 \mu \mathrm{l}$, the column temperature $45{ }^{\circ} \mathrm{C}$, and the wavelength $205 \mathrm{~nm}$. The concentrations of individual flavonoids and triterpenoids were determined by comparison with external standards. Total flavonoids and total triterpenoids were the sum of individuals.

\section{Environmental factors}

Site data were collected from the collection sites with a GPS (JUNO®SCSD, Trimble, Sunnyvale, CA, USA), including longitude, latitude, and altitude. Annual mean 
temperatures, rainfall, sunlight, and number of frost-free days were obtained from the nearest meteorological station or from the National Meteorological Data Service Centre of China (https://data.cma.cn/data/detail/dataCode/SURF_ CLI_CHN_MUL_DAY_CES_V3.0.html) that provides the closest approximated values for each region.

\section{Statistical analyses}

Means and standard deviations (SD) were calculated for each flavonoid and triterpenoid content. Correlations analyses between environmental variables and flavonoid and triterpenoid contents were performed separately. The analyses were conducted using SPSS statistics software 19.0. Initial principal component analyses (PCAs) were carried out using flavonoid and triterpenoid contents as the variable factors to describe data variation characteristics. Additional PCAs were undertaken to examine the relationship between high flavonoid individuals, high triterpenoid individuals, and environmental factors. According to the PCA analyses and correlation analyses, the environment factors associated with secondary metabolite variations were selected, described by scatter plots, and tested by paired t-tests. All PCA analyses were conducted in the $\mathrm{R}$ v. 3.4.4 statistical environment ( $\mathrm{R}$ Development Core Team 2018) with the package vegan $\mathrm{v}$. 1.8-5 (Oksanen et al. 2007). Scatter plots and paired t-tests were conducted using the SPSS statistics software 19.0.

\section{Results}

\section{Geographic distribution}

A total of 343 C. paliurus accessions from 32 distinct populations were found across the $C$. paliurus distribution range in southern China (Fig. 1; Table 1). The geographic distribution ranged from Henan province to Guangxi province, and from Zhejiang province to Guizhou province, showing that C. paliurus is widely adapted. However, there are preferred growth features and habitats: (1) dominant or subdominant in competition; (2) growing in valleys or on limestone hills with an altitude limit of 400-1770 m; and, (3) scattered in relatively small populations in mixed forest.

The altitude of $C$. paliurus ranged from $400 \mathrm{~m}$ a.s.l. (Anhui) to $1770 \mathrm{~m}$ a.s.1. (Guangxi). Mean annual rainfall ranged from $714 \mathrm{~mm}$ (Shanxi) to $2119 \mathrm{~mm}$ (Zhejiang), and annual mean temperatures from $11.3{ }^{\circ} \mathrm{C}$ (Hubei) to $18.1^{\circ} \mathrm{C}$ (Fujian). Annual hours of sunlight ranged from $968 \mathrm{~h}$ (Sichuan) to $1969 \mathrm{~h}$ (Anhui), while the number of frost-free days ranged from $219 \mathrm{~d}$ (Henan) to $357 \mathrm{~d}$ (Guangxi).

\section{Secondary metabolites in C. paliurus leaves}

This study primarily focused on flavonoid and triterpenoid variations and chemical traits identified by HPLC according to Cao et al. (2017), including quercetin-3-O-glucuronide, quercetin-3-O-galactoside, isoquercitrin, kaempferol-3-Oglucuronide, kaempferol 3-O-glucoside, quercetin-3-Orhamnoside, kaempferol-3-O-rhamnoside, arjunolic acid, cyclocaric acid B, pterocaryoside B, pterocaryoside A, hederagenin, and oleanolic acid.

To analyze their variations, the maximum, minimum, and average amounts of flavonoids and triterpenoids were calculated together with their respective standard deviations (Table 2). These findings indicate that most flavonoid and triterpenoid contents varied greatly. Overall, triterpenoids (1.7-44.5 mg/g) showed relatively higher variation than flavonoids (0.6-27.1 mg/g). Among the flavonoids, quercetin-3-O-glucuronide (0-6.0 mg/g) and kaempferol-3-Oglucuronide $(0.1-6.9 \mathrm{mg} / \mathrm{g})$ varied widely, with the means being $1.8 \mathrm{mg} / \mathrm{g}$ and $1.4 \mathrm{mg} / \mathrm{g}$, respectively. Of the triterpenoids, arjunolic acid $(0.2-11.5 \mathrm{mg} / \mathrm{g})$, pterocaryoside B (0-19.2 mg/g), and pterocaryoside A (0.1-16.4 mg/g) showed significantly wide variations with average values of $3.4 \mathrm{mg} / \mathrm{g}, 2.7 \mathrm{mg} / \mathrm{g}$ and $3.6 \mathrm{mg} / \mathrm{g}$, respectively. One interesting result is that the relative $\mathrm{CV}$ (coefficient of variation) for isoquercitrin (1.4) and kaempferol 3-O-glucoside (1.4) are much higher than for the other flavonoids and triterpenoids.

Table 2 Flavonoid and triterpenoid compositions (mg/g) in 343 C. paliurus individual leaves

\begin{tabular}{|c|c|c|c|c|c|c|c|c|c|c|c|}
\hline Flavonoids & Min & Max & Mean & SD & $\mathrm{CV}$ & Triterpenoids & Min & Max & Mean & $\mathrm{SD}$ & $\mathrm{CV}$ \\
\hline Quercetin-3-O-glucuronide & 0.0 & 6.0 & 1.8 & 1.3 & 0.7 & Arjunolic acid & 0.2 & 11.5 & 3.4 & 2.0 & 0.6 \\
\hline Quercetin-3-O-galactoside & 0.0 & 4.4 & 0.6 & 0.4 & 0.8 & Cyclocaric acid B & 0.3 & 6.4 & 1.5 & 1.1 & 0.7 \\
\hline Isoquercitrin & 0.0 & 8.4 & 0.4 & 0.6 & 1.4 & Pterocaryoside B & 0.0 & 19.2 & 2.7 & 2.9 & 1.1 \\
\hline Kaempferol-3-O-glucuronide & 0.1 & 6.9 & 1.4 & 0.9 & 0.6 & Pterocaryoside A & 0.1 & 16.4 & 3.6 & 2.7 & 0.8 \\
\hline Kaempferol 3-O-glucoside & 0.0 & 4.0 & 0.4 & 0.6 & 1.4 & Hederagenin & 0.0 & 4.7 & 1.0 & 0.7 & 0.7 \\
\hline Quercetin-3-O-rhamnoside & 0.0 & 1.2 & 0.2 & 0.2 & 0.7 & Oleanolic acid & 0.1 & 1.5 & 0.4 & 0.2 & 0.6 \\
\hline Kaempferol-3-O-rhamnoside & 0.0 & 4.8 & 1.5 & 1.0 & 0.6 & Total triterpenoid & 1.7 & 44.5 & 12.6 & 7.0 & 0.6 \\
\hline Total flavonoid & 0.6 & 27.1 & 6.3 & 3.4 & 0.5 & & & & & & \\
\hline
\end{tabular}

$\mathrm{SD}=$ standard deviation. Zero indicate that the compound concentration is lower than detection limit 


\section{Correlation analyses}

As shown in Table 3, correlation analyses showed flavonoids and triterpenoids with limited correlation to three geographical and three climate factors. Of these, latitude was negatively correlated with arjunolic acid $(R=-0.368, P<0.05)$ content while longitude was negatively correlated with isoquercitrin levels $(R=-0.374, P<0.05)$. Altitude was negatively correlated with total triterpenoid levels $(R=-0.364$, $P<0.05)$ and pterocaryoside A content $(R=-0.360$, $P<0.05)$. For climate factors, average annual temperatures were negatively correlated with kaempferol-3-O-glucuronide $(R=-0.43, P<0.05)$; annual precipitation was positively correlated with pterocaryoside $\mathrm{A}(R=0.390, P<0.05)$ and negatively with isoquercitrin $(R=-0.384, P<0.05)$ and kaempferol-3-O-glucoside $(R=-0.426, P<0.05)$. The number of annual frost-free days was negatively correlated with hederagenin $(R=-0.391, P<0.05)$.

Based on the analyses, there were moderate correlations at $P<0.05$ significance level and the maximum absolute value of the correlation coefficient was weak at 0.426 . Overall, the correlation between $C$. paliurus leaf flavonoids and triterpenoids and environmental factors appears to be very limited. While considering correlation analyses at a macro-scale might lead to the loss of information, a deeper analysis of individual flavonoid and triterpenoid variations was required.

\section{Principal component analysis (PCA)}

A PCA biplot analyzed the data of $C$. paliurus leaf flavonoids and triterpenoids (Fig. 2). The first two PCAs, analyzed from 15 variables of flavonoids and triterpenoids, explained $55.0 \%$ of the total variation (PC 1 explained $31.4 \%$ and PC 2 explained 23.6\%). Most flavonoid and triterpenoid

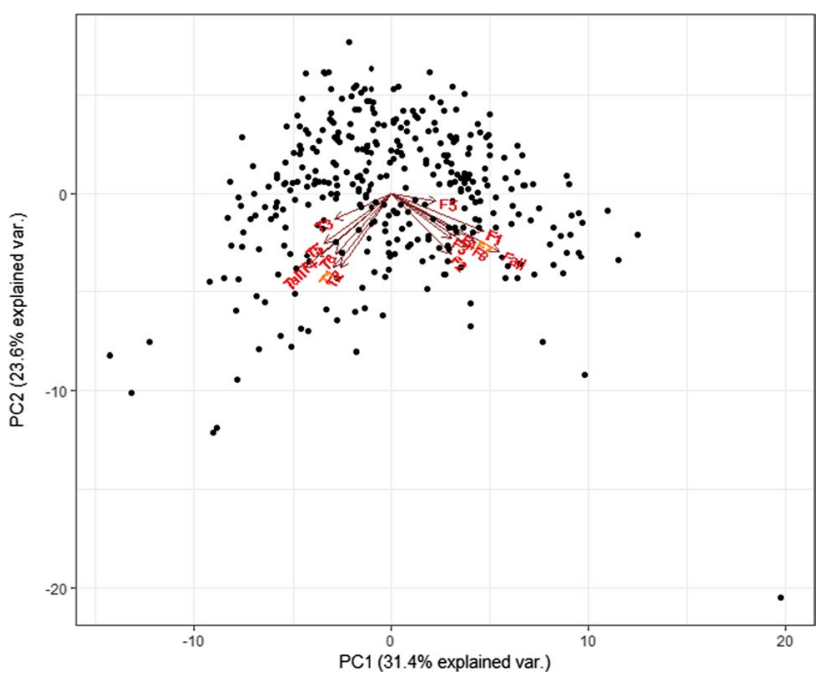

Fig. 2 Principal component analysis of $C$. paliurus flavonoids and triterpenoids; T1: arjunolic acid, T2: cyclocaric acid B, T3: pterocaryoside B, T4: pterocaryoside A, T5: hederagenin, T6: oleanolic acid, Tall: total triterpenoid, F1: quercetin-3-O-glucuronide, F2: quercetin-3-O-galactoside, F3: isoquercitrin, F4: kaempferol-3-O-glucuronide, F5: kaempferol 3-O-glucoside, F6: quercetin-3-O-rhamnoside, F7: kaempferol-3-O-rhamnoside, and Fall: total flavonoid
Table 3 Correlation analysis results between environmental factors and leaf traits

\begin{tabular}{lrllccrc}
\hline & Lat & Lon & \multicolumn{1}{l}{ Alt } & Te & Prec & Sundur & Ff \\
\hline Arjunolic acid & $-0.368^{*}$ & 0.095 & -0.335 & 0.231 & 0.287 & 0.032 & 0.125 \\
Cyclocaric acid B & -0.277 & 0.146 & -0.275 & 0.199 & 0.249 & 0.021 & 0.118 \\
Pterocaryoside B & -0.203 & 0.121 & -0.224 & -0.035 & 0.211 & -0.029 & -0.06 \\
Pterocaryoside A & -0.208 & 0.296 & $-0.360^{*}$ & 0.042 & $0.390^{*}$ & -0.004 & -0.132 \\
Hederagenin & 0.269 & 0.345 & -0.206 & -0.186 & 0.044 & 0.253 & $-0.391^{*}$ \\
Oleanolic acid & 0.164 & 0.317 & -0.236 & -0.06 & 0.121 & 0.102 & -0.309 \\
Total triterpenoid & -0.258 & 0.235 & $-0.364^{*}$ & 0.076 & 0.336 & 0.023 & -0.063 \\
Quercetin-3-O-glucuronide & 0.183 & -0.005 & 0.085 & -0.346 & 0.095 & 0.012 & -0.068 \\
Quercetin-3-O-galactoside & -0.071 & 0.034 & -0.112 & 0.183 & -0.04 & 0.149 & 0.084 \\
Isoquercitrin & 0.169 & $-0.374 *$ & 0.233 & 0.07 & $-0.384^{*}$ & -0.084 & 0.071 \\
Kaempferol-3-O-glucuronide & 0.319 & -0.114 & 0.195 & $-0.403^{*}$ & -0.076 & -0.109 & -0.18 \\
Kaempferol 3-O-glucoside & 0.284 & -0.285 & 0.226 & 0.103 & $-0.426^{*}$ & -0.032 & -0.011 \\
Quercetin-3-O-rhamnoside & 0.156 & -0.144 & 0.068 & -0.124 & -0.04 & -0.178 & -0.033 \\
Kaempferol-3-O-rhamnoside & 0.088 & -0.263 & 0.103 & -0.021 & -0.073 & -0.197 & 0.163 \\
Total flavonoid & 0.282 & -0.232 & 0.192 & -0.21 & -0.164 & -0.089 & -0.013 \\
\hline
\end{tabular}

* Significant correlation at the $P<0.05$ level

Alt: altitude, Te: annual average temperature, Prec: average annual precipitation, Lat: latitude, Lon: longtitude, Sundur: sunshine duration, and Ff: frost-free days 
information was included, especially for total triterpenoid (0.99) and total flavonoid (0.98) content (Table 4). Information for cyclocaric acid B (0.77), pterocaryoside B (0.89), pterocaryoside A (0.82), quercetin-3-O-glucuronide $(0.82)$, Isoquercitrin (0.78), and kaempferol-3-O-glucoside (0.75) was also included. Based on the results, two principal component analysis adequately described the data structure of C. paliurus leaf flavonoids and triterpenoids.

As shown in Fig. 2, different types of flavonoids showed almost the same direction of variation, indicating that they changed in basically the same pattern, as did the triterpenoids. However, flavonoids and triterpenoids showed different directions in variation but it appeared that they interacted to some extent. According to Figs. 2 and 3, the resources could be divided into three groups: group 1 with high flavonoid and low triterpenoid contents; group 2 with high triterpenoid and low flavonoid contents; and, group 3 with low flavonoid and low triterpenoid contents. In other words, flavonoids and triterpenoids suppressed each other when either levels increased. With the total flavonoid content being more than $10 \mathrm{mg} / \mathrm{g}, 45$ high flavonoid (HFla) individuals were divided, including 12 from Sichuan, 10 from Hubei, 5 from Anhui, 4 from Shanxi, 3 from Jiangxi, 4 from Guangxi, one from Guizhou, 4 from Zhejiang, and one from Fujian. Twenty high triterpenoid (HTri) individuals (levels more than $25 \mathrm{mg} / \mathrm{g}$ ) were divided, including 12 from Jiangxi, 4 from Hunan, and one each from Henan, Anhui, Guizhou and Guangxi.

To determine the extent to which environmental factors affect secondary metabolites, additional PCAs were carried out (Fig. 4). The first two, generated from six environmental variables, including latitude, longitude, altitude, annual average temperatures, sunshine duration, and annual frost-free days explained $74.9 \%$ of all variations. The PCA model extracted most of the environmental factor information of HFla, HTri, and normal

Table 4 Paired t-tests of environmental factors according to secondary metabolite contents

\begin{tabular}{llccl}
\hline Pairs & $\begin{array}{l}\text { Environmental } \\
\text { factors }\end{array}$ & No. & $\begin{array}{l}\text { Correlation } \\
\text { coefficient }\end{array}$ & $P$ value \\
\hline HTri-LTri & Altitude & 32 & 0.399 & $0.024^{*}$ \\
HFla-LFla & Latitude & 32 & -0.304 & 0.091 \\
HFla-LFLa & longitude & 32 & 0.307 & 0.087 \\
HFla-HTri & longitude & 25 & 0.357 & 0.079 \\
HFla-HTri & Altitude & 25 & -0.514 & $0.009^{* *}$ \\
HFla-HTri & Rainfall & 25 & 0.416 & $0.039^{*}$ \\
\hline
\end{tabular}

*Significant correlation at the $P<0.05$ level,**Extremely significant correlation at the $P<0.01$ level

HTri: locations with high triterpenoid individuals, LTri: locations with low triterpenoid individuals, HFla: locations with high flavonoid individuals, LFla: locations with low flavonoid individuals

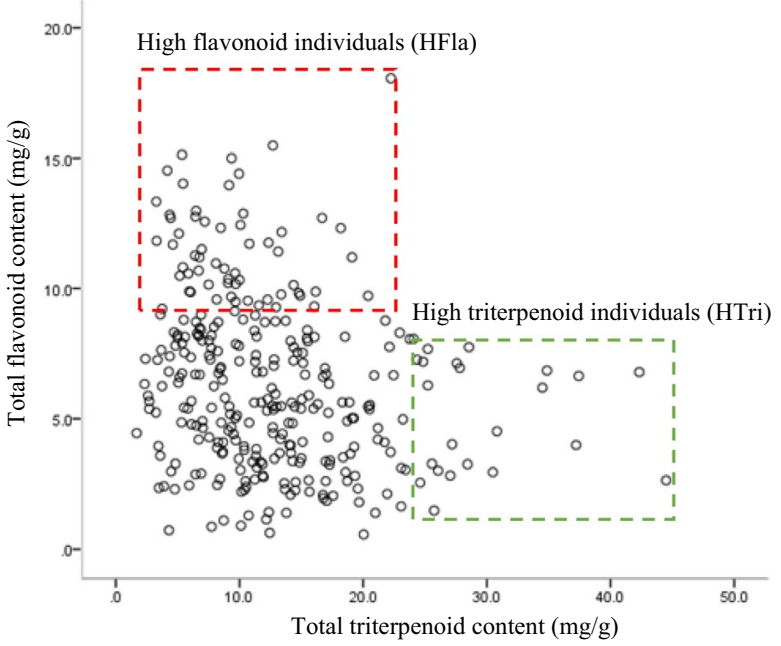

Fig. 3 Analysis of C. paliurus germplasm flavonoids and triterpenoids $(\mathrm{mg} / \mathrm{g})$

individuals. Sites with more HFla individuals (Fig. 4) were mostly distributed in higher latitudes or at higher altitudes, while sites with more HTri individuals were mostly distributed in areas with higher rainfall. This confirms that environmental factors play a role in secondary metabolite variation. When comparing the environmental factors

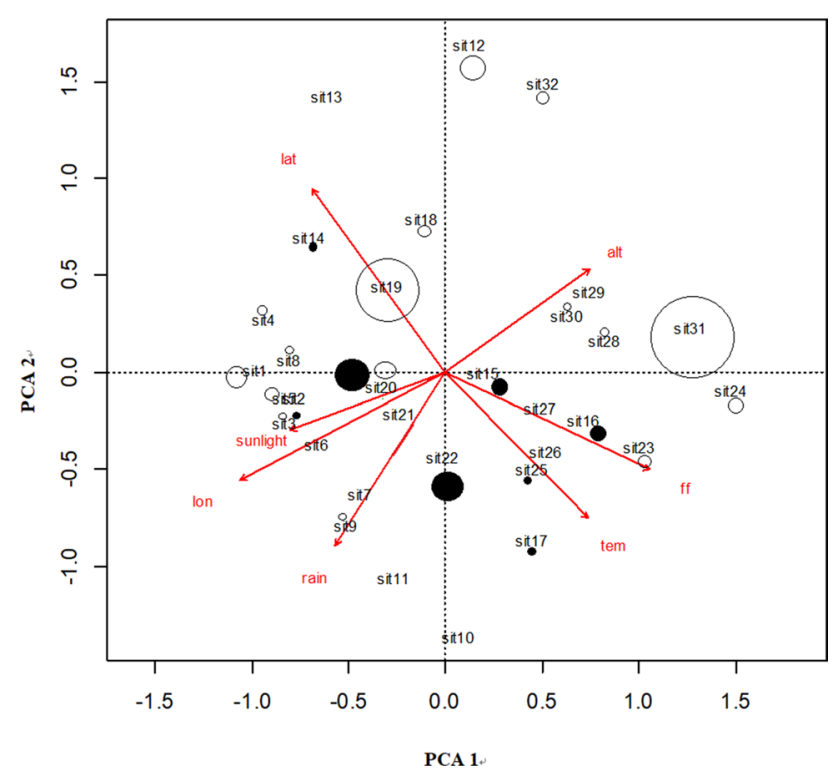

Fig. 4 Principal component analysis of environmental factors of $C$. paliurus germplasm location. lat: latitude, alt: altitude, lon: longitude, tem: annual average temperature, rain: average annual precipitation, sunlight: sunshine duration and ff: frost-free days; sit indicates population locations. Open circle indicate locations with high-flavonoid (HFla) individuals, the larger area the more individuals. Filled circle indicate locations with high-triterpenoid (HTri) individuals, the larger area the more individuals 
associated with the distribution of HFla and HTri individuals together, the results were not totally symmetrical; there was an overlap that consisted of part of the scattering area of the HTri individuals and the whole of the scattering area of the HFla individuals (Fig. 4).

T-tests were conducted to identify any significant environmental differences between individuals with more secondary metabolites and normal individuals (Table 4). The analysis was carried out between environmental factors of HTri and LTri individuals, HFla and LFla individuals, and HTri and HFla individuals (Fig. 3). Notably, compared with HTri locations, HFla locations were significantly negatively correlated with altitude, with correlation coefficients as high as -0.514 . Moreover, rainfall was also significantly positively correlated between HTri locations and HFla locations at the $P<0.05$ level, with the correlation coefficient being 0.416 .

From the PCA and t-test results, scatter plots were developed to examine the geographical variations in secondary metabolites more closely. As shown in Fig. 5a, HTri individuals were distributed with longitude, ranging from almost $108^{\circ} \mathrm{E}$ to $118^{\circ} \mathrm{E}$, and across altitudes from sea level to $1000 \mathrm{~m}$. However, HFla individuals were generally scattered in the northwest portion of the $C$. paliurus range (Fig. 5b). In addition, compared with HFla individuals (Fig. 5c), HTri individuals were scattered across lower altitudes from sea level to $1000 \mathrm{~m}$, while HFla individuals were relatively insensitive to altitude (from sea level to $2000 \mathrm{~m}$ ). Taken together, these results indicate that secondary metabolites were definitely affected by environmental factors, but flavonoid and triterpenoid synthesis were not completely opposed.

\section{Discussion}

Genetic and environmental factors and their interactions are known to affect plant secondary metabolite synthesis (Hamilton et al. 2001); the mechanism underlying this process requires further investigation. According to the carbon-nutrient balance hypothesis $(\mathrm{CNBH})$, production of phytochemical defense substances is limited by the available carbon and nitrogen nutrients in plant tissues. Massad et al. (2012) found that flavonoids in Pentaclethra macroloba (Willd.) Kuntze seedling leaves fit the CNBH while triterpenoids did not. They attributed this to different biosynthetic pathways of the two secondary metabolites. Wang et al. (2017b) found that the triterpenoid saponin, and the total flavonoid content of Glycyrrhiza uralensis Fisch adventitious roots were synergistically enhanced significantly by the combination of methyl jasmonate and phenylalanine, indicating an interaction between flavonoid and triterpenoid synthesis. According to the analysis in this study, because of the different accumulating directions between flavonoids and triterpenoids, the $C$. paliurus leaves were divided into high triterpenoid, high flavonoid and normal plants, while no leaves with high contents of both were found. Alqahtani et al. (2015) considered that, in the herbaceous Centella asiatica (L.) Urban, flavonoid compounds and triterpenes showed similar patterns influenced by the time of collection. Our results suggest, however, that when stimulated by environment factors, only either flavonoids or triterpenoids increased but not both, contrary to Alqahtani et al. (2015) and Wang et al. (2017b). Interaction between flavonoids and triterpenoids in $C$. paliurus leaves may be associated with environmental conditions or trade-offs between growth and defense (Keinänen et al. 1999; Laitinen et al. 2005). From a

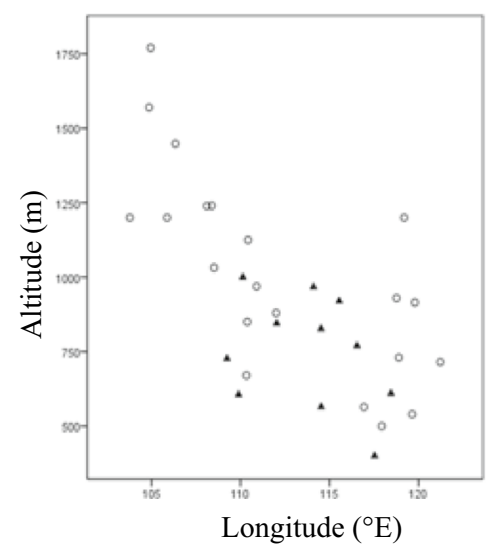

(a)

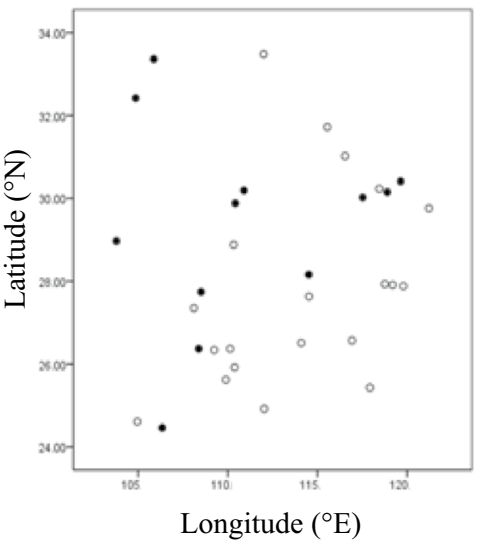

(b)

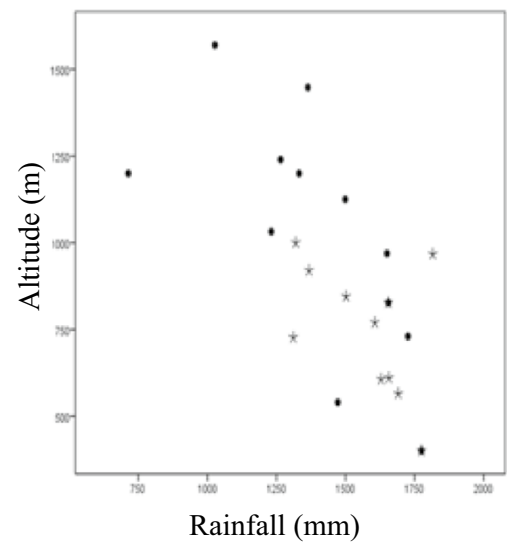

(c)
Fig. 5 Environment characteristics of high-triterpenoid (HTri) individuals and high-flavonoid (HFla) individuals; a Filled triangle indicate locations of high-triterpenoid (HTri) individuals and Open circle locations of other (LTri) individuals. b Filled circle indicate loca- tions of high-flavonoid (HFla) individuals and Open circle locations of other (LFla) individuals. c Filled circle indicate locations of highflavonoid (HFla) individuals and * indicate high-triterpenoid (HTri) individuals 
commercial perspective, C. paliurus leaves should be studied in two directions, ones with high flavonoids and ones with high triterpenoid contents.

Previous studies have analyzed the responses of secondary metabolites to abiotic factors (Deng et al. 2015, 2018; Liu et al. 2018), and phenotypic variations in secondary metabolite accumulation (Fang et al. 2011; Cao et al. 2017, 2018) of C. paliurus. While almost all genotypic investigations have been carried out at the population level (Deng et al 2015, 2017, 2019a, b; Liu et al. 2016), Cao et al. (2018) recognized this and considered that there were large variations between individuals in secondary metabolite contents. Our research is in agreement with Cao et al. (2018) and has analyzed to the individual level, in case there was information missed at the population level, including genotypic, microclimatic, and soil aspects.

According to previous findings, environmental stress often significantly influences flavonoid synthesis in plants, especially stress caused by UV radiation and low temperatures (Kootstra et al. 1994; Casati and Walbot 2003; Valkama et al. 2004; Stracke et al. 2010). Jaakola and Hohtola (2010) found that northern climates, possibly because of long days of sunlight and cool night temperatures, had a positive impact on flavonoid biosynthesis. This effect remained obvious, in spite of variations between species and within individual groups. In addition, Liu et al. (2016) found that the flavonoid contents of $C$. paliurus leaves were highly positively correlated with total solar radiation. Our results show that the northwestern part of the $C$. paliurus range at high latitudes or at higher altitudes contained almost all the high-flavonoid individuals. Compared with the study of Deng et al. (2015), no significant environmental factors were identified in specific ranges, while a general trend was found in the whole $C$. paliurus population. This is in agreement with Jaakola and Hohtola (2010) and suggests that areas with higher altitudes or latitudes provided sufficient solar radiation and cool night temperatures to stimulate flavonoid synthesis. This is also consistent with Liu et al. (2016) who found that $C$. paliurus leaves from Muchuan County had the highest flavonoid content. Our results suggest that highflavonoid $C$. paliurus resources should be investigated from the northwest areas with higher altitudes.

Park et al. (2005) and Schlag and Mclntosh (2006) found that the same species growing in different environments generally had differences in triterpenoid variety and content. Fan et al. (2013) and Sun et al. (2018) found that higher temperatures in the range of soapberry (Sapindus mukorossi Gaertn.) resulted in higher yields of the triterpenoid saponin. Jiao and Gao (2011) and Zeng et al. (2012) also reported that temperature significantly influenced the synthesis of the triterpenoid saponin. Most studies agree that triterpenoid synthesis is affected more significantly by environment than genotype. In C. paliurus however Deng et al. (2018), found that individual triterpenoids could be influenced by genotype while the environment influenced the total triterpenoid variation. Our study included more sites and so allowed a comparison of the environmental factors between high triterpenoid plants and other individuals. This is in partial agreement with Deng et al. (2018), however, we found that altitude was significantly correlated with $C$. paliurus triterpenoid content with high triterpenoid individuals scattered almost solely on sites under $1000 \mathrm{~m}$ and below longitudes of $117^{\circ}$ N. Based on Deng et al. (2018), this suggests that lower altitudes and rainfall might promote triterpenoid synthesis across the whole distribution area. It was also found that high triterpenoid individuals require relatively higher precipitation and lower altitudes, while high flavonoid plants are distributed across a broader range of conditions. The results agreed with a study of balsam pear that showed that higher relative humidity promotes total saponins and inhibits total flavonoids ( $\mathrm{Li}$ et al. 2015). Higher temperatures and photosynthetically active radiation promote flavonoid accumulations but are unfavorable for saponin synthesis (Li et al. 2015).

We found that environmental factors influenced accumulation when the level of one kind of secondary metabolite was much higher than the other. However, this only occurred at the family level and not at the population level in each location, implying that potential genotypic or environmental aspects might be involved as indicated previously (Deng et al. 2015, 2017). This is in agreement with Deepak et al. (2018) who found that genotype-related secondary metabolite variations (between families or populations) were much stronger than provenance in silver birch. Genotypes, even between families, differ in their responses to the environment (Keinänen et al. 1999; Laitinen et al. 2005), so the combination of different genotypes and their environments may produce high secondary metabolite content. Tree age and vigor, the age of leaves, soil conditions, and microclimate might also play a role in this process. In this study, the key environmental factors and distribution correlated with high secondary metabolite accumulation were identified.

\section{Conclusions}

Environmental factors that affect flavonoid and triterpenoid levels were identified and this information will be valuable for the selection of suitable $C$. paliurus cultivation areas and directed breeding. Further research should examine interactions between genetic and environmental factors in secondary metabolite synthesis to help identify mechanisms underlying secondary metabolite variations of this economically important species. 
Acknowledgments We are grateful to Drs. Wanxia Yang, Bo Deng, and Zhang Leying, and Mr Qingliang Liu from the Nanjing Forestry University for field and laboratory assistance. We also thank Dr. Emma Tacken from Liwen Bianji, Edanz Group China (www.liwenbianji.cn. ac) for editing the English text of a draft of this manuscript.

Open Access This article is licensed under a Creative Commons Attribution 4.0 International License, which permits use, sharing, adaptation, distribution and reproduction in any medium or format, as long as you give appropriate credit to the original author(s) and the source, provide a link to the Creative Commons licence, and indicate if changes were made. The images or other third party material in this article are included in the article's Creative Commons licence, unless indicated otherwise in a credit line to the material. If material is not included in the article's Creative Commons licence and your intended use is not permitted by statutory regulation or exceeds the permitted use, you will need to obtain permission directly from the copyright holder. To view a copy of this licence, visit http://creativecommons.org/licenses/by/4.0/.

\section{References}

Alqahtani A, Tongkao-on W, Li KM, Razmovski-Naumovski V, Chan K, Li GQ (2015) Seasonal variation of triterpenes and phenolic compounds in Australian Centella asiatica (L.) Urb. Phytochem Anal 26(6):436-443

Barnes PW, Flint SD, Ryel RJ, Tobler MA, Barkley AE, Wargent JJ (2015) Rediscovering leaf optical properties: new insights into plant acclimation to solar UV radiation. Plant Physiol Biochem 93:94-90

Cao YN, Fang SZ, Yin ZQ, Fu XX, Shang XL, Yang WX, Yang HM (2017) Chemical fingerprint and multicomponent quantitative analysis for the quality evaluation of Cyclocarya paliurus leaves by HPLC-Q-TQF-MS. Molecules 22(11):1927-1942

Cao YN, Deng B, Fang SZ, Shang XL, Fu XX, Yang WX (2018) Genotypic variation in tree growth and selected flavonoids in leaves of Cyclocarya paliurus. South For 80(1):67-74

Casati P, Walbot V (2003) Gene expression profiling in response to ultraviolet radiation in maize genotypes with varying flavonoid content. Plant Physiol 132(4):1739-1754

Deepak M, Lihavainen J, Keski-Saari S, Kontunen-Soppela S, Salojarvi J, Tenkanen A, Heimonen K, Oksanen E, Keinanen M (2018) Genotype- and provenance-related variation in the leaf surface secondary metabolites of silver birch. Can J For Res 48(5):494-505

Deng B, Shang XL, Fang SZ, Li QQ, Fu XX, Su J (2012) Integrated effects of light intensity and fertilization on growth and flavonoid accumulation in Cyclocarya paliurus. J Agric Food Chem 60:6286-6292

Deng B, Fang SZ, Yang WX, Tian Y, Shang XL (2014) Provenance variation in growth and wood properties of juvenile Cyclocarya paliurus. New For 45:625-639

Deng B, Cao YN, Fang SZ, Shang XL, Yang WX, Qian CY (2015) Variation and stability of growth and leaf flavonoid content in Cyclocarya paliurus across environments. Ind Crop Prod 76:386-393

Deng B, Fang S, Shang X, Fu X, Li Y (2017) Influence of provenance and shade on biomass production and triterpenoid accumulation in Cyclocarya paliurus. Agroforest Syst 1:1-10

Deng B, Fang S, Shang X, Fu X, Yang W (2018) Influence of genotypes and environmental factors on leaf triterpenoid content and growth of Cyclocarya paliurus. J For Res 30:789-798
Deng B, Fang SZ, Shang XL, Fu XX, LiY Y (2019a) Influence of provenance and shade on biomass production and triterpenoid accumulation in Cyclocarya paliurus. Agroforest Syst 93:483-492

Deng B, Fang SZ, Shang XL, Fu XX, Yang WX (2019b) Influence of genotypes and environmental factors on leaf triterpenoid content and growth of Cyclocarya paliurus. J For Res 30(3):789-798

Fan HH, Yao XM, Zhang TY, Lin XQ, Tang XH, Ma L (2013) The selection of high-saponin-yield plus tree of Sapindus mukorossi. J For Sci Technol 40:69-73

Fang SZ, Fu XX (2007) Progress and prospects on silviculture and utilization of Cyclocarya paliurus resources. J Nanjing For Univ 31(1):95-100

Fang SZ, Wang JY, Wei ZY, Zhu ZX (2006) Methods to break seed dormancy in Cyclocarya paliurus (Batal.) Iljinskaja. Sci HorticAmst 110(3):305-309

Fang SZ, Yang WX, Chu XL, Shang XL, She CQ, Fu XX (2011) Provenance and temporal variations in selected flavonoids in leaves of Cyclocarya paliurus. Food Chem 124:1382-1386

Hamilton G, Zanger AR, DeLucia EH, Berenbaum MR (2001) The carbon nutrient balance hypothesis: its rise and fall. Ecol Lett 4:86-95

Jaakola L, Hohtola A (2010) Effect of latitude on flavonoid biosynthesis in plants. Plant Cell Environ 33(8):1239-1247

Jiao XL, Gao WW (2011) Advances in studies on influence of environmental factors on triterpenoid saponin synthesis in medicinal plants. Chin Tradit Herb Drugs 42(2):398-402

Keinänen M, Julkunen-Tiitto R, Mutikainen P, Walls M, Ovaska J, Vapaavuori E (1999) Trade-offs in phenolic metabolism of silver birch: effects of fertilization, defoliation, and genotype. Ecology 80(6):1970-1986

Kootstra A (1994) Protection from UV-B-induced DNA damage by flavonoids. Plant Mol Biol 26(2):771-774

Kurihara H, Asami S, Shibata H, Fukami H, Tanaka T (2003) Hypolipemic effect of Cyclocarya paliurus (Batal) Iljinskaja in lipid-loaded mice. Biol Pharm Bull 26:383-385

Laitinen ML, Julkunen-Tiitto R, Tahvanainen J, Heinonen J, Rousi M (2005) Variation in birch (Betula pendula) shoot secondary chemistry due to genotype, environment, and ontogeny. J Chem Ecol 31(4):697-717

Li WP, Liang YL, Bao TL, Mu L, Gao DK (2015) Responses of total flavonoids and total saponins contents in fruits and leaves of balsam pear to soil moisture and their correlations. Food Sci 36(8):134-138

Li QQ, Hu JL, Xie JH, Nie SP, Xie MY (2017) Isolation, structure, and bioactivities of polysaccharides from Cyclocarya paliurus (Batal.) Iljinskaja. Ann NY Acad Sci 1398(1):20-29

Li XC, Fu XX, Shang XL, Yang WX, Fang SZ (2017) Natural population structure and genetic differentiation for heterodicogamous plant: Cyclocarya paliurus, (Batal.) Iljinskaja (Juglandaceae). Tree Genet Genom 13(4):80-89

Liu Y, Qian CY, Ding SH, Shang XL, Yang WX, Fang SZ (2016) Effect of light regime and provenance on leaf characteristics, growth and flavonoid accumulation in Cyclocarya paliurus (Batal) Iljinskaja coppices. Bot Stud 57(1):28

Liu Y, Cao YN, Fang SZ, Wang TL, Yin ZQ, Shang XL, Yang WX, Fu XX (2018) Antidiabetic effect of Cyclocarya paliurus leaves depends on the contents of antihyperglycemic flavonoids and antihyperlipidemic triterpenoids. Molecules 23(5):1042-1058

Massad TJ, Dyer LA, Vega CG (2012) Costs of defense and a test of the carbon-nutrient balance and growth-differentiation balance hypotheses for two co-occurring classes of plant defense. PLoS ONE 7(10):e47554

Oksanen J, Kindt R, Legendre P, O'Hara B (2007) Vegan: Community Ecology Package. R Foundation for Statistical Computing, Vienna, Austria 
Park JD, Rhee DK, Lee YH (2005) Biological activities and chemistry of saponins from Panax ginseng C. A Meyer Phytochem Rev 4:159-175

R Development Core Team (2007) R: a language and environment for statistical computing. R Foundation for Statistical Computing, Vienna, Austria

Schlag EM, McIntosh MS (2006) Ginsenoside content and variation among and within American ginseng (Panax quinquefolius L.) populations. Phytochem 67:1510-1519

Shang XL, Wu ZF, Yin ZQ, Zhang J, Liu ZJ, Fang SZ (2015) Simultaneous determination of flavonoids and triterpenoids in Cyclocarya paliurus leaves using high-performance liquid chromatography. Afr J Tradit Compl 12(3):125-134

Shu RG, Xu CR, Li LN, Yu ZL (1995) Cyclocariosides II and III: two secodammarane triterpenoid saponins from Cyclocarya paliurus. Planta Med 61(5):551-553

Shu RG, Xu CR, Li LN (1995) Studies on the sweet principles from the leaves of Cyclocarya paliurus (Batal.) Iljinsk. Acta Pharmaceut 30:757-761

Stracke R, Favory JJ, Gruber H, Bartelniewoehner L, Bartels S, Binkert M, Funk M, Weisshaar B, Ulm R (2010) The Arabidopsis bZIP transcription factor HY5 regulates expression of the PFG1/ MYB12 gene in response to light and ultraviolet-B radiation. Plant Cell Environ 33(1):88-103

Sun CW, Wang LC, Liu JM, Zhao GC, Gao SL, Xi BY, Duan J, Weng XH, Jia LM (2018) Genetic structure and biogeographic divergence among Sapindus species: An inter-simple sequence repeatbased study of germplasms in China. Ind Crop Prod 118:1-10

Szakiel A, Paczkowski C, Henry M (2011) Influence of environmental abiotic factors on the content of saponins in plants. Phytochem Rev 10:471-491

Valkama E, Salminen JP, Koricheva J, Pihlaja K (2004) Changes in leaf trichomes and epicuticular flavonoids during leaf development in three birch taxa. Ann Bot 94(2):233-242
Wang QQ, Jiang CH, Fang SZ, Wang JH, Ji Y, Shang XL, Ni YC, Yin ZQ, Zhang J (2017) Antihyperglycemic, antihyperlipidemic and antioxidant effects of ethanol and aqueous extracts of Cyclocarya paliurus leaves in type 2 diabetic rats. J Ethnopharmacol 150(3):1119-1127

Wang J, Li J, Wu XL, Liu SJ, Li HF, Gao WY (2017) Assessment of genetic fidelity and composition: Mixed elicitors enhance triterpenoid and flavonoid biosynthesis of Glycyrrhiza uralensis Fisch tissue cultures. Biotechnol Appl Biochem 64(2):211-217

Wright M, Byrd J, Gao Y, Stubblefield J, Park H, Dunlap N (2014) Isolation and structural clarification of triterpenes from Cyclocarya paliurus: Cyclocaric acid A and B. Planta Med 80:139-143

Xie MY, Li L, Nie SP, Wang XR, Lee FSC (2006) Determination of speciation of elements related to blood sugar in bioative extracts from Cyclocarya paliurus leaves by FIA-ICP-MS. Eur Food Res Technol 223(2):202-209

Xie JH, Dong CJ, Nie SP, Li F, Wang ZJ, Shen MY, Xie MY (2015) Extraction, chemical composition and antioxidant activity of flavonoids from Cyclocarya paliurus (Batal.) Iljinskaja leaves. Food Chem 186(4):97-105

Zeng Y, Guo L, Yang G, Chen B, Wang J, Huang L (2012) Effect of environmental ecological factors on saponins of medicinal plant. Chin J Exp Tradit Med Formulae 18(17):313-318

Zhou MM, Lin Y, Fang SZ, Liu Y, Shang XL (2019) Phytochemical content and antioxidant activity in aqueous extracts of Cyclocarya paliurus leaves collected from different populations. Peer J 7:e6492

Publisher's Note Springer Nature remains neutral with regard to jurisdictional claims in published maps and institutional affiliations. 